\title{
Patients Age is a Critical Factor in Determining the Severity of Osteoarthritis of the Shoulder
}

\section{Nicole Märtens ( $\nabla$ nicole.maertens@med.ovgu.de )}

Otto-von-Guericke-Universitat Magdeburg Medizinische Fakultat

\section{Vincent Maerz}

Medizinische Hochschule Hannover

\section{Jessica Bertrand}

Otto-von-Guericke-Universitat Magdeburg Medizinische Fakultat

Christoph H Lohmann

Otto-von-Guericke-Universitat Magdeburg Medizinische Fakultat

\section{Alexander Berth}

Otto-von-Guericke-Universitat Magdeburg Medizinische Fakultat

\section{Research article}

Keywords: osteoarthritis, shoulder, arthroplasty, OARSI, Constant-Murley-Score, age, pain

Posted Date: August 21st, 2020

DOI: https://doi.org/10.21203/rs.3.rs-58692/v1

License: (c) (i) This work is licensed under a Creative Commons Attribution 4.0 International License. Read Full License 


\section{Abstract}

Background:

Osteoarthritis (OA) is one of the most common musculoskeletal disorders in the aging population. The correlation of radiographic $\mathrm{OA}$ severity, disability and pain is variable and inconsistent for the different joints. This study aims to elucidate the relationship between histological and radiological signs of shoulder OA with pain sensation and functional impairment to potentially adapt the recommendation for surgical treatment for primary total shoulder arthroplasty (TSA).

Methods:

Forty-four patient with shoulder OA undergoing a TSA using an anatomic stemless implant were included in this study. The radiological OA severity was scored preoperatively on true ap X-rays according to the Kellgren-Lawrence-Score (KL-Score). The histological OA severity was determined according to the OARSIScore using bone-cartilage sections from loaded areas of the humeral head. Pain was quantified with the Visual Analog Scale (VAS). The functional status was assessed by the items "mobility" and "strength" out of the Constant-Murley-Score (CS-Score).

Results:

There was no correlation between radiographic and histological severity in shoulder OA. However, a correlation between age and the severity of radiological changes was observed. Further, pain did not correlate with histological or radiological scores, whereas it correlated with age. The functional shoulder status (mobility, strength) correlated with the severity of radiological changes, but not with the histologic scoring.

Conclusion:

This study shows that increased age is the main determinant of radiological changes in shoulder $O A$, as well as pain. Therefore, age and pain sensation should be considered as important parameters for the recommendation for TSA.

\section{Background}

Osteoarthritis $(\mathrm{OA})$ is one of the most common age related diseases. ${ }^{10} \mathrm{OA}$ is characterized by progressive loss of cartilage, new bone formation, and inflammation of the synovial tissue. ${ }^{2}$ Clinically, OA can be diagnosed by the radiographic evidence of cartilage damage and it is considered to cause increased complaints about joint pain, restricted activities of daily living, loss of function and strength of the affected joint. ${ }^{23}$ The Kellgren-Lawrence-Score (KL-Score), therefore, has been proposed as one radiographic diagnostic method to estimate the extent of shoulder OA. ${ }^{16}$ Therefore, OA can also be approached by assessing subjective and objective determinants of the respective joint. 
A correlation between structural damage and radiological appearance for hip and knee OA has been clearly shown previously. ${ }^{31,32}$ Additionally, some studies showed a correlation between pain and radiological OA severity. ${ }^{3,14}$ This correlation, however, is controversially discussed in the literature for both, the knee and the hip joint. ${ }^{4,12}$ One study showed, that an increased radiological score in the knee joint was associated with increased pain frequency scores. ${ }^{9}$ Another study showed that no significant association between radiographic lesions and pain frequency could be proven for the hip, giving rise to the assumption that the pain sensation might be joint specific. ${ }^{25}$

$\mathrm{OA}$ of the shoulder joint is less frequent than hip or knee OA. ${ }^{2,23}$ Furthermore, there are even less information about the correlation between radiographic changes and pain for the shoulder joint. The optimal management of primary shoulder $\mathrm{OA}$ is a topic of ongoing investigation. ${ }^{1}$ Beside conservative treatment, numerous surgical techniques exist including non-arthroplasty techniques and anatomic or reversed shoulder replacement systems. ${ }^{1}$ Although primary total shoulder arthroplasty (TSA) has been accepted as an effective and secure procedure to relieve pain and to restore shoulder function in patients with end-stage $\mathrm{OA}$, who failed to conservative treatment, it is potentially associated with a number of complications. ${ }^{8,20,30}$ Therefore, the indication for TSA needs to be proven carefully. Besides a justified indication for TSA considering the respective joint pathology, an accurate surgical technique with utilization of an adequate implant and the appropriate patient selection are essential factors for a successful outcome of TSA.

The aim of this study was to elucidate the relation between radiological changes and histological appearance of OA with pain sensation as well as the functional status in patients with shoulder OA. This aims to provide more insight to amend the recommendations for TSA in patients with shoulder OA.

\section{Methods}

\section{Patients}

This study included 44 patients (19 male / 25 female, $68.5 \pm 17.5$ years) with primary OA of the shoulder treated with a stemless humeral head replacement between December 2011 and May 2013. Informed consent obtained from all patients enrolled in the study after IRB approval of the faculty of medicine. (IRB No: $55 / 11)$.

\section{Radiographic analysis}

Standardized X-ray images in true anterior-posterior view in neutral position of the forearm of the patients were performed preoperatively. The KL-Score was used for scoring of shoulder OA severity. ${ }^{16}$ As we did not include instability of the shoulder joint as well as rotator cuff arthropathy in our study, we decided to use the KL-Score instead of the Samilson- or Hamada Fukuda-Score. The KL-Score is mainly based on the quantification of osteophytes, joint space narrowing and subchondral bone sclerosis. These are the 
key features we investigated in this study. All radiographs were reviewed by two independent observers and a consensus score was generated during a meeting.

\section{Histological analysis}

OA grade was evaluated using a histology score (OARSI-Score). Briefly, the resected humeral head were cut into quarter slices (centre to periphery). All specimens were fixed in $4 \%$ PFA for $24 \mathrm{~h}$, decalcified, and embedded in paraffin. 3-4 $\mu \mathrm{m}$ sections were cut and stained using Safranin-Orange. OARSI Score was used to assess the histological osteoarthritis score was used (grades $0-6$ ). ${ }^{26}$ The classification OARSI scoring for of each blinded sample, which was reviewed by two independent observers and a consensus score was generated during a meeting..

\section{Clinical Evaluation}

One of the authors clinically examined all patients before and after TSA procedure. The functional status of the patients was assessed with the items "mobility" and "strength" accordingly to the Constant MurleyScore. ${ }^{6}$ The pain sensation of each patient was assessed using a visual analog scale (VAS; 0 - no pain, 15 - max. pain).

\section{Statistical analysis}

Statistical analysis performed using GraphPad 6 Software (La Jolla California, USA). The statistical significance was analysed using a linear regression. Statistical significance was considered for a $p$-value $<0.05$.

\section{Results}

\section{Description of the patient cohort}

The pre-operative functional joint status of the patients was measured using the Constant-Murley (CS) Score with an average of $13.2 \pm 7.4$ points for "mobility" and $2.6 \pm 2.3$ points for "strength" in the total cohort. The pre-operative pain sensation of the patients was measured using the VAS-Pain score (8.7 \pm 2.6 points). The radiographic evaluation of the true anteroposterior $X$-rays showed a grade 1 in 5 patients, a grade 2 in 10 patients, a grade 3 in 6 patients and a grade 4 in 23 patients according to the KL-Score.

\section{No correlation between radiological and histological OA grade}


Additionally, we scored cartilage-bone sections from the main loaded area of the humeral head using the OARSI-Score. When comparing the radiographic OA severity with histological signs of OA, there were patients with severe radiographic and histological OA (figure 1a). Interestingly, we also observed patients with low radiographic OA severity, but marked histological OA severity (figure 1b). However, we also found patients with severe radiographic OA and mild OA like cartilage changes (figure 1c). The classification of the histological OA grade of the specimens according to the OARSI-Score demonstrates grade 1 in 1 patient, a grade 2 in 3 patients, a grade 3 in 13 patients and a grade 4 in 6 patients, grade 5 in 11 patients and grade 6 in 7 patients. In line with the above mentioned observations, we found no correlation in the anterior-posterior projection in KL classification and OARSI scoring for comparing the grade of shoulder $O A(p=0.4)$ (figure 1d).

\section{Correlation between pain and OA severity}

To understand whether there is a correlation between pain and the radiographic OA severity, the KL-Score was plotted against the values from the VAS-Pain-Scale specific for the shoulder for each patient. There was no significant correlation between KL-Score and VAS-Pain-Scale $(p=0.2)$ (figure 2a). Furthermore, there was no correlation between the VAS-Pain-Scale and the OARSI-Score $(p=0.4)$ (figure $2 b)$ in the tested cohort. Interestingly, a correlation was found when comparing age with pain $(p=0.04)$ (figure $2 c)$ for each patient. This finding indicates that older patients had significantly more pain compared to younger patients.

\section{Joint function correlated with KL-Score, but not with histological assessment}

Furthermore, we tested the influence of radiological and histological signs of OA on the joint function. The correlation analysis between the KL-Score and the functional status of the shoulder joint, represented via the items "mobility" and "strength", was significant ( $p=0.0003, p=0.04$ respectively) (figure 3a, b). However, the items "mobility" and "strength" did not correlate with the histological OARSI-Score $(p=0.6$ and $p=0.8$, respectively) (figure $3 c, 3 d$ ).

\section{Age correlated with Kellgren-Lawrence Score, but not OARSI-Score}

Since OA proposed to be an age related disease, we investigated the correlation between radiological signs of OA using the KL-Score and age. We observed a strong correlation between both parameter $(p=0.03)$ (figure 4a). A correlation analysis between the histological OA grade and the age of the respective patient, revealed no correlation between both parameters $(p=0.8)$ (figure $4 b$ ). 


\section{Discussion}

The aim of the study was to investigate the interaction between typical clinical findings in patients with shoulder OA who failed to conservative treatment in consideration to the indication of primary TSA. Therefore, correlation analysis performed including the parameters pain, functional involvement, radiological and histological appearance and age in patients undergoing a stemless humeral head replacement.

The findings of the present study lead to the assumption that pain sensation correlates with patient age. Furthermore, our data show a correlation between severity of radiographic changes of arthritic shoulder joint and the functional impairment of the involved shoulder. The characteristic histologic evaluation of structural cartilage damage in shoulder OA does not seem to be directly associated with the radiographic severity as well with the limitations in function and pain sensation of the affected arthritic joint (overview in suppl. table 1).

The success of TSA as a treatment option in patients with shoulder OA is depending on many factors. ${ }^{11}$ Objective parameters such as the improvement of range of motion and an increase of strength as well as subjective values like pain reduction are involved in patient satisfaction after surgery. ${ }^{15,27}$ The observation that the radiographic $O A$ signs increase with increasing patient age is in line with a previous study. ${ }^{17}$ However, the missing correlation between radiographic OA changes and histological OA changes has not been described so far. To our knowledge, only one study has analysed the relationship of signs of OA e.g. the hyaline articular cartilage calcification with age and histologic OA grade using the OARSI-Score before. ${ }^{13}$ In line with our findings, the authors did not observe a correlation between OARSIScore and age. However, the study did not include the KL-Score or the VAS-Pain-Score of the patients since this study included only post-mortem samples. The phenomenon that the histological OARSI-Score correlates with the radiographic changes has been described for hand and knee OA before. ${ }^{31,32} \mathrm{~A}$ possible explanation for this discrepancy in the shoulder joint might be due to limitations of grading osteochondral lesions with $\mathrm{x}$-ray radiographs utilizing the KL-Score, which was not originally applied to the glenohumeral joint. ${ }^{16}$ Furthermore, shoulder $\mathrm{OA}$ is often associated with a posterior humeral head dislocation. The size and location of resulting osteochondral lesions ("posterior wear") may be not detectable using X-ray analyses. ${ }^{28}$

The data of the present study suggest that neither the radiographic nor the histological joint changes had a significant influence on the pain sensation in patients with OA. This observation is in line with a previous study also showing that the radiographic changes in the shoulder do not correlate with the VASPain-Score. ${ }^{18}$ In contrast, other studies show a correlation between pain intensity and KL-Score for other joints e.g. the knee ${ }^{3,22}$ or hip. ${ }^{14}$ These incongruent results within several joints lead to the assumption that pain intensity and disease severity in radiographs may differ for each joint of the body. This discrepancy may also be explained by some aspects of the pathology of OA that are related to pain like synovitis or bone marrow edema that cannot be seen in X-ray imaging. ${ }^{24}$ Additionally, periarticular 
pathologies such as subacromial bursitis and / or rotator cuff - and biceps tendinitis are also common in patients with shoulder OA resulting in local pain and not depicted in x-ray pictures. These numerous "nonarticular pain conditions" might also contribute to the discrepancy between pain intensity and the radiographic severity in the shoulder $\mathrm{OA} .{ }^{24}$

The average age in this study population is in the typical range for OA. ${ }^{29}$ In this context the present study shows, that the age of the patient correlated with pain intensity estimated by a VAS score. This finding indicates that age-related effects might influence pain sensation in shoulder OA. Several studies have evaluated the impact of age as a risk factor for pain perception. ${ }^{19} 21,34$ Interestingly, animal studies demonstrated that the sensitization of nociceptors to mechanical stimulation depended on age and the chronicity of the inflammation. ${ }^{20,31}$ Moreover, human OA pain sensation is a complex phenomenon and beside structural articular pathologies like cartilage alterations or inflammation due to synovitis it is caused and modulated by various other factors, e.g. central pain pathways and genetic factors. $7,33,35$

This study also shows that the functional status of the shoulder, evaluated according to the CS-Score items "mobility" and "strength" are clearly associated with the severity of the radiographic articular changes. This might be explained by the fact that a decreased joint volume, a tight capsule, osteophytes and the deformation of the articular surface may cause a mechanical restriction of the joint motion as well as a reduced ability to raise and hold a specific weight in a given position. However, the histopathological evidence of glenohumeral cartilage degeneration, did not seem to affect shoulder function and pain sensation as shown in this present study. It is well known that in contrast to osteochondral defects of weight-bearing joints such as the hip, knee, and ankle, even large degenerative glenohumeral cartilaginous lesions can be well tolerated. ${ }^{5}$ As the shoulder is a non-weight-bearing joint, a strong correlation between the extent of cartilage damage, pain intensity and functional disability is lacking.

We are well aware of the limitation of our study, which includes only 44 patients. More patients are needed to unravel the complex relations between structural joint damage and pain sensation.

\section{Conclusion}

The diagnosis and treatment options of shoulder OA are commonly based on functional impairment and radiographic signs of $\mathrm{OA}$. This study shows that particularly pain intensity and patient age are important parameters in supporting treatment plans for shoulder $\mathrm{OA}$ and should be taken into account for decision making in TSA. However, further investigations are needed to evaluate the exact impact of the structural pathology, functional limitations and pain for the recommendation of TSA in patients with shoulder OA.

\section{Abbreviations}

Total shoulder arthroplasty (TSA); Osteoarthritis (OA); Constant Murley Score (CS); Kellgren and Lawrence Score (KL Score); Histology score (OARSI Score); visual analog scale pain score (VAS pain score) 


\section{Declarations}

\section{Ethics approval and consent to participate}

The IRB of the medical faculty (Ethik -Kommission der Otto-von-Guericke Universität an der Medizinischen Fakultät und am Universitätsklinikum A.ö.R. Univ. Prof. Dr.med. C. Huth) approved the study (IRB No: 55/11). Written informed consent was obtained from all patients before including them in the study.

\section{Consent for publication}

Not applicable.

\section{Availability of data and materials}

The data that support the findings of this study are available from the authors, Data are however available from the authors upon reasonable request.

\section{Competing interests}

Professor Christoph H. Lohmann is a paid consultant for the following companies Waldemar Link GmbH \& Co. KG, Mathys Medical $\mathrm{GmbH}$ and ImplanTec $\mathrm{GmbH}$ and has royalties from ImplanTec $\mathrm{GmbH}$. Dr. Alexander Berth is a paid consultant for Mathys Medical $\mathrm{GmbH}$. The authors confirm that there has been no significant financial support for this work that could have influenced its outcome.

\section{Funding}

This manuscript received funding from the DFG (BE 4328/9-1). The DFG paid for consumables and partially paid the technician involved in cutting of the samples.

\section{Authors' contributions}

NM performed all experiments, VM enrolled the patients and measured the VAS-Score, JB helped with the evaluation of the OARSI Score and writing of the manuscript, $\mathrm{CHL}$ helped writing of the manuscript and evaluation of the clinical data, Ab identified the patients and evaluated all data and wrote the manuscript. All authors have read and approved the manuscript.

\section{Acknowledgements}


The authors acknowledge the excellent technical assistance of Carolin Schneider, Anja Schröder and Mandy Könnecke.

\section{References}

1. Ansok CB, Muh SJ. Optimal management of glenohumeral osteoarthritis. Orthop Res Rev 2018 Feb 23;10:9-18. doi: 10.2147/ORR.S134732. eCollection;\%2018.:9-18.

2. Bertrand J, Cromme C, Umlauf D, Frank S, Pap T. Molecular mechanisms of cartilage remodelling in osteoarthritis. Int J Biochem Cell Biol. 2010 Oct;42(10):1594-601.

3. Cho HJ, Chang CB, Yoo JH, Kim SJ, Kim TK. Gender differences in the correlation between symptom and radiographic severity in patients with knee osteoarthritis. Clin Orthop Relat Res. 2010 Jul;468(7):1749-58.

4. Cicuttini FM, Baker J, Hart DJ, Spector TD. Association of pain with radiological changes in different compartments and views of the knee joint. Osteoarthritis Cartilage. 1996 Jun;4(2):143-7.

5. Cole BJ, Yanke A, Provencher MT. Nonarthroplasty alternatives for the treatment of glenohumeral arthritis. J Shoulder Elbow Surg. 2007 Sep;16(5 Suppl):231-40.

6. Constant CR, Murley AH. A clinical method of functional assessment of the shoulder. Clin Orthop Relat Res 1987 Jan;(214):160-4.

7. Deveza LA, Melo L, Yamato TP, Mills K, Ravi V, Hunter DJ. Knee osteoarthritis phenotypes and their relevance for outcomes: a systematic review. Osteoarthritis Cartilage. 2017 Dec;25(12):1926-41.

8. Duan X, Zhang W, Dong X, Liu M, Gao Y, Huang F, et al. Total shoulder arthroplasty versus hemiarthroplasty in patients with shoulder osteoarthritis: a meta-analysis of randomized controlled trials. Semin Arthritis Rheum. 2013 Dec;43(3):297-302.

9. Duncan R, Peat G, Thomas E, Hay E, McCall I, Croft P. Symptoms and radiographic osteoarthritis: not as discordant as they are made out to be? Ann Rheum Dis 2007 Jan;66(1):86-91.

10. Felson DT. Developments in the clinical understanding of osteoarthritis. Arthritis Res Ther. 2009;11(1):203.

11. Friedman RJ, Eichinger J, Schoch B, Wright T, Zuckerman J, Flurin PH, et al. Preoperative parameters that predict postoperative patient-reported outcome measures and range of motion with anatomic and reverse total shoulder arthroplasty. JSES Open Access 2019 Nov 18;3(4):266-72.

12. Hannan MT, Felson DT, Pincus T. Analysis of the discordance between radiographic changes and knee pain in osteoarthritis of the knee. J Rheumatol. 2000 Jun;27(6):1513-7.

13. Hawellek T, Hubert J, Hischke S, Vettorazzi E, Wegscheider K, Bertrand J, et al. Articular cartilage calcification of the humeral head is highly prevalent and associated with osteoarthritis in the general population. J Orthop Res. 2016 Nov;34(11):1984-90.

14. lidaka T, Muraki S, Akune T, Oka H, Kodama R, Tanaka S, et al. Prevalence of radiographic hip osteoarthritis and its association with hip pain in Japanese men and women: the ROAD study. Osteoarthritis Cartilage. 2016 Jan;24(1):117-23. 
15. Jacobs CA, Morris BJ, Sciascia AD, Edwards TB. Comparison of satisfied and dissatisfied patients 2 to 5 years after anatomic total shoulder arthroplasty. J Shoulder Elbow Surg. 2016 Jul;25(7):112832.

16. Kellgren JH, Lawrence JS. Radiological Assessment of Osteo-Arthrosis. Ann Rheum Dis. 1957;16:494-501.

17. Kircher J, Kuerner K, Morhard M, Krauspe R, Habermeyer P. Age-related joint space narrowing independent of the development of osteoarthritis of the shoulder. Int J Shoulder Surg. 2014 Oct;8(4):95-100.

18. Kircher J, Morhard M, Magosch P, Ebinger N, Lichtenberg S, Habermeyer P. How much are radiological parameters related to clinical symptoms and function in osteoarthritis of the shoulder? Int Orthop. 2010 Jun;34(5):677-81.

19. Lautenbacher S, Peters JH, Heesen M, Scheel J, Kunz M. Age changes in pain perception: A systematic-review and meta-analysis of age effects on pain and tolerance thresholds. Neurosci Biobehav Rev 2017 Apr;75:104-113. doi: 10.1016/j.neubiorev.2017.01.039. Epub;\%2017 Jan 31.:104 $-13$.

20. Mattei L, Mortera S, Arrigoni C, Castoldi F. Anatomic shoulder arthroplasty: an update on indications, technique, results and complication rates. Joints 2015 Nov 3;3(2):72-7.

21. McDougall JJ, Schuelert N. Age alters the ability of substance $P$ to sensitize joint nociceptors in guinea pigs. J Mol Neurosci. 2007;31(3):289-96.

22. Muraki S, Akune T, Teraguchi M, Kagotani R, Asai $Y$, Yoshida $M$, et al. Quadriceps muscle strength, radiographic knee osteoarthritis and knee pain: the ROAD study. BMC Musculoskelet Disord 2015 Oct 16;16:305. doi: 10.1186/s12891-015-0737-5.:305-0737.

23. Neer CS. Replacement arthroplasty for glenohumeral osteoarthritis. J Bone Joint Surg Am. 1974;56:1-13.

24. O'Neill TW, Felson DT. Mechanisms of Osteoarthritis (OA) Pain. Curr Osteoporos Rep. 2018 Oct;16(5):611-6.

25. Pereira D, Severo M, Santos RA, Barros $H$, Branco J, Lucas $R$, et al. Knee and hip radiographic osteoarthritis features: differences on pain, function and quality of life. Clin Rheumatol. 2016 Jun;35(6):1555-64.

26. Pritzker KP, Gay S, Jimenez SA, Ostergaard K, Pelletier JP, Revell PA, et al. Osteoarthritis cartilage histopathology: grading and staging. Osteoarthritis Cartilage. 2006 Jan;14(1):13-29.

27. Schoch BS, King JJ, Wright TW, Vigan M, Werthel JD. Defining the tipping point for primary shoulder arthroplasty. JSES Open Access 2019 Nov 18;3(4):273-7.

28. Schumaier A, Abboud J, Grawe B, Horneff JG, Getz C, Romeo A, et al. Evaluating Glenohumeral Osteoarthritis: The Relative Impact of Patient Age, Activity Level, Symptoms, and Kellgren-Lawrence Grade on Treatment. Arch Bone Jt Surg. 2019 Mar;7(2):151-60.

29. Shane AA, Loeser RF. Why is osteoarthritis an age-related disease? Best Pract Res Clin Rheumatol. 2010 Feb;24(1):15-26. 
30. Singh JA, Sperling J, Buchbinder R, McMaken K. Surgery for shoulder osteoarthritis: a Cochrane systematic review. J Rheumatol. 2011 Apr;38(4):598-605.

31. Stove J, Gerlach C, Huch K, Gunther KP, Brenner R, Puhl W, et al. Gene expression of stromelysin and aggrecan in osteoarthritic cartilage. Pathobiology. 2001;69(6):333-8.

32. Sunk IG, Amoyo-Minar L, Niederreiter B, Soleiman A, Kainberger F, Smolen JS, et al. Histopathological correlation supports the use of $x$-rays in the diagnosis of hand osteoarthritis. Ann Rheum Dis. 2013 Apr;72(4):572-7.

33. Warner SC, Walsh DA, Laslett LL, Maciewicz RA, Soni A, Hart DJ, et al. Pain in knee osteoarthritis is associated with variation in the neurokinin 1/substance P receptor (TACR1) gene. Eur J Pain. 2017 Aug;21(7):1277-84.

34. Weyer AD, Zappia KJ, Garrison SR, O'Hara CL, Dodge AK, Stucky CL. Nociceptor Sensitization Depends on Age and Pain Chronicity(1,2,3). eNeuro 2016 Feb 8;3(1):eN-0115.

35. Woolf CJ. Central sensitization: implications for the diagnosis and treatment of pain. Pain 2011 Mar;152(3 Suppl):S2-15.

\section{Figures}



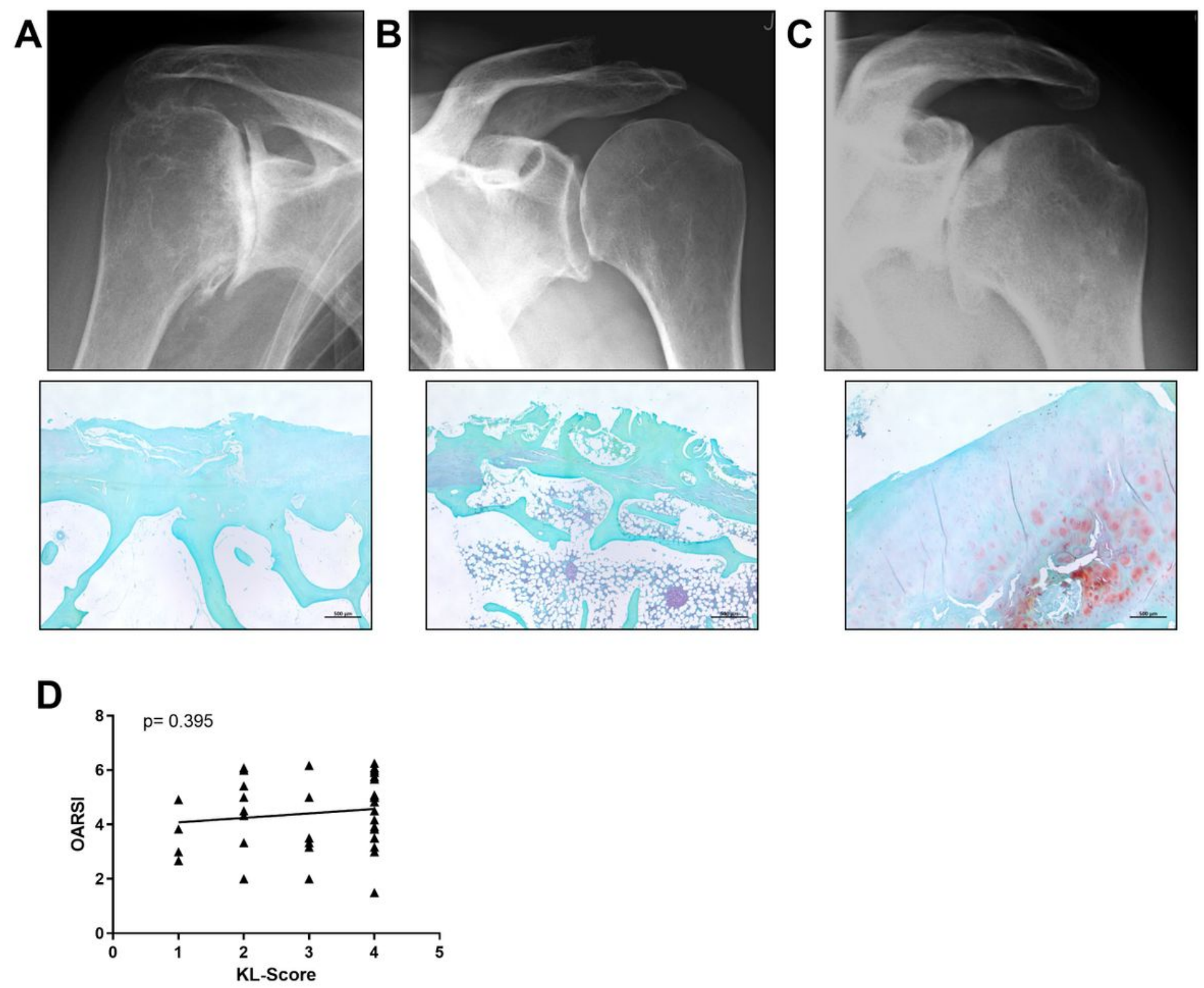

\section{Figure 1}

No correlation between radiological and histological OA grade A) Representative true anteroposterior Xray picture with- Kellgren-Lawrence grade 4and corresponding histological cartilage evaluation (OARSI 5). B) True anteroposterior X-ray picture with KL grade 2 and corresponding histology (OARSI 6). C) True anteroposterior X-ray with KL grade 4 and corresponding histology (OARSI 4) D) Correlation between OARSI Score. 
A

B
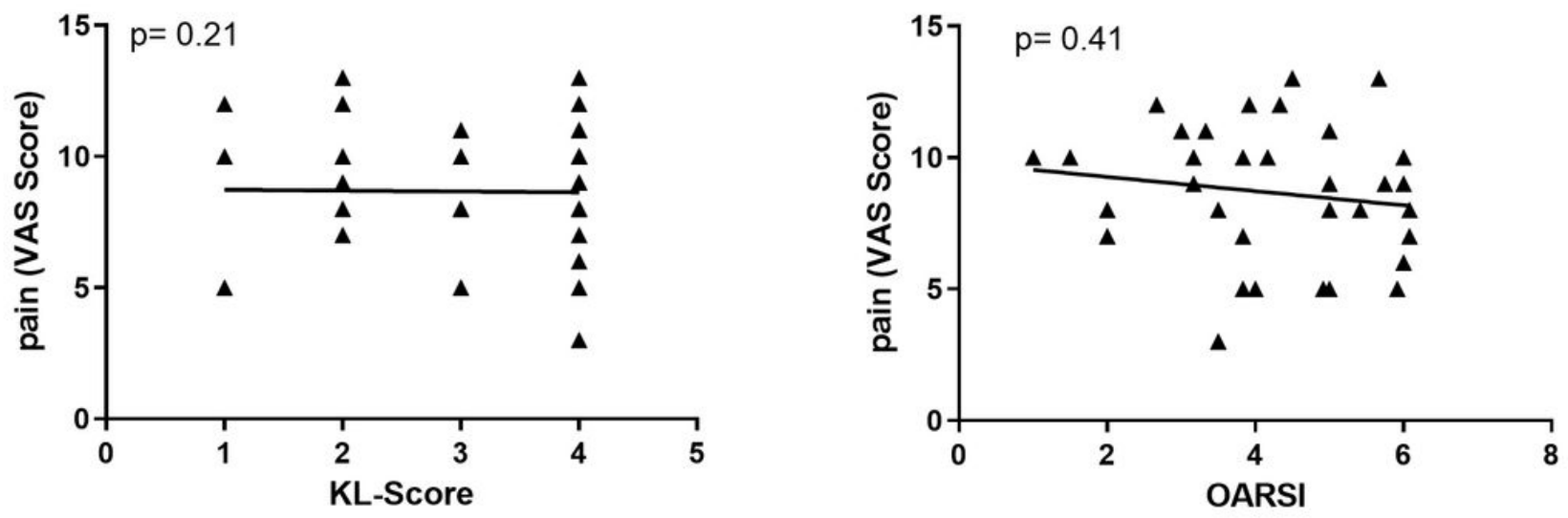

C

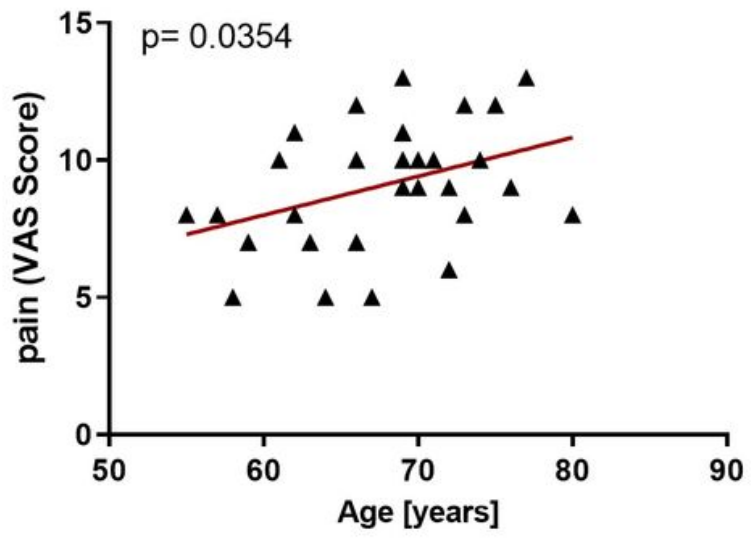

Figure 2

No correlation between radiological and histological OA grade. A) Linear regression analysis of pain (VAS-Score) data with Kellgren-Lawrence Score $(N=33, p=0.21) B$ ) Linear regression analysis of pain (VAS-Score) with OARSI score $(\mathrm{N}=31, \mathrm{p}=0.41) \mathrm{C}$ ) Liner regression analysis of pain (VAS-Score) with age $(\mathrm{N}=31, \mathrm{p}=0.0354)$. 
A
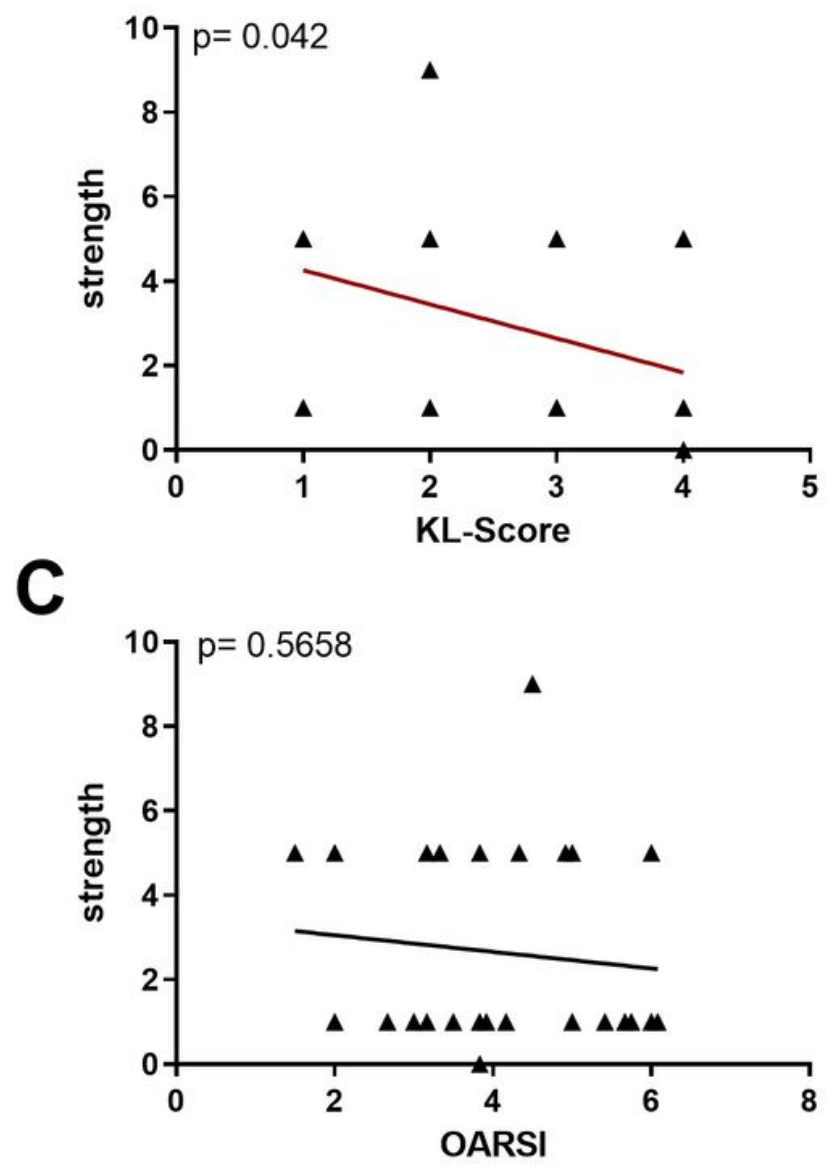

B

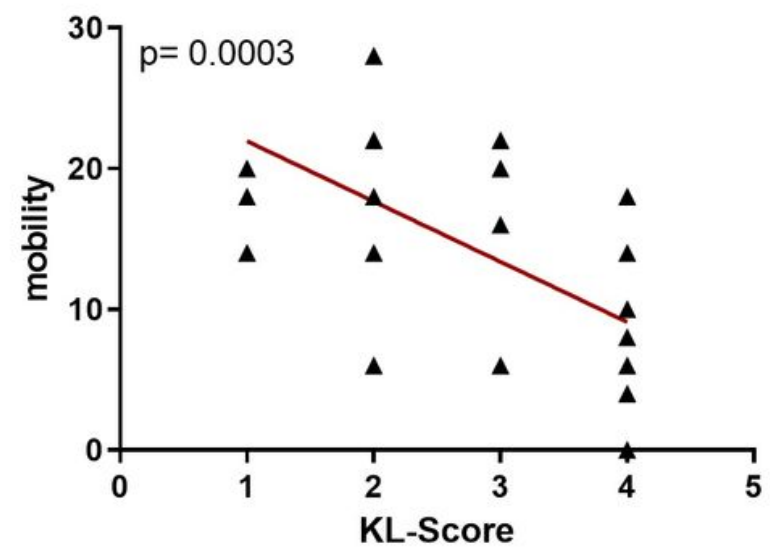

D

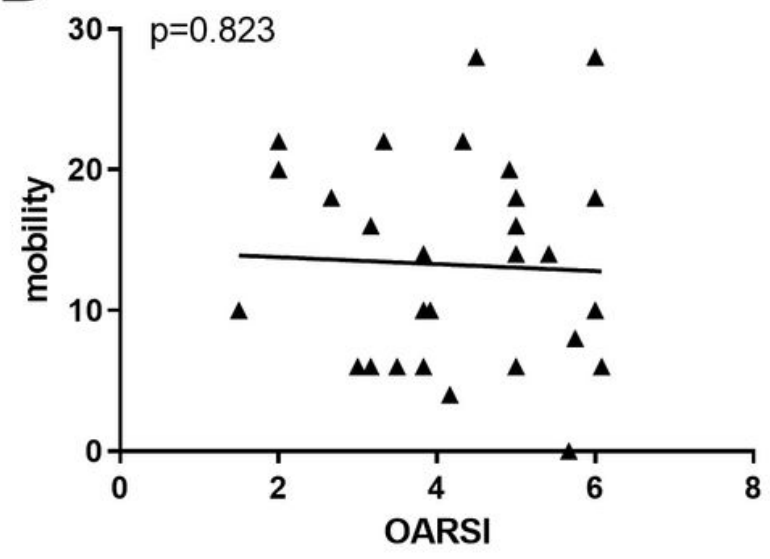

Figure 3

Joint function correlated with KL-Score, but not histological assessment. A) Linear regression analysis of the Constant-Murley Score item "strength" and "mobility (B) with radiological changes $(N=31, p=0.042$ and $p=0.003$, respectively. $C$ ) Linear regression analysis of the Constant-Murley-Score item "strength" and "mobility" (D) with the histological OARSI score ( $N=31, p=0.5558$ and 0.823 , respectively). 
A

B
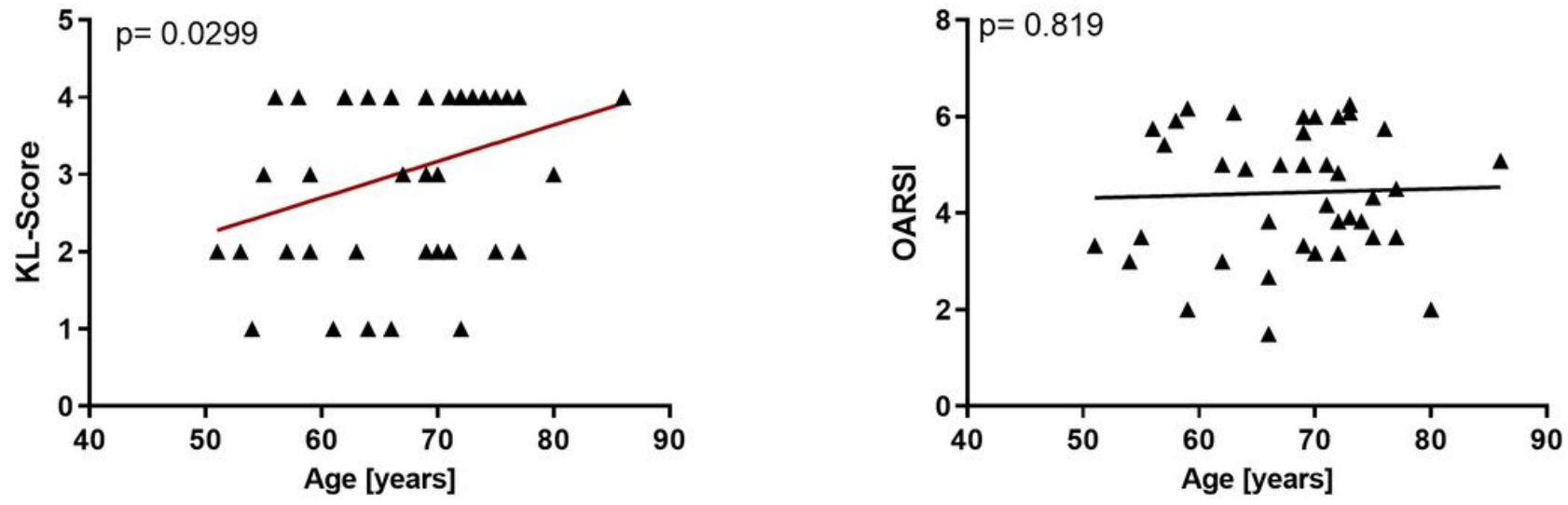

Figure 4

Age correlated with Kellgren-Lawrence Score, but not OARSI-Score. A) KL-Score correlation with age (N= $44, p=0.0299)$ B) OARSI score correlation with age $(N=41, p=0.819)$

\section{Supplementary Files}

This is a list of supplementary files associated with this preprint. Click to download.

- Table1.docx 